\title{
Prescribing Patterns and Use of Risk-Reduction Tools After Implementing an Opioid-Prescribing Protocol
}

\author{
Matthew A. Breeden, MD, Christine K. Jacobs, MD, Matthew Witthaus, MD, \\ Joanne Salas, MPH, Kelly M. Everard, PhD, Eric Penton, BS, and \\ Jeffrey F. Scherrer, $P h D$
}

Background: The literature on results from primary care-based opioid-prescribing protocols is small and results have been mixed. To advance this field, we evaluated whether opioid prescribing changed after a comprehensive protocol was implemented and whether change was associated with the number and type of risk reduction tools adopted.

Methods: Electronic medical record data were obtained for 2607 patients. Demographics, Patient Health Questionnaire-9 scores, body mass index, and utilization levels of protocol elements were measured for 24 months prior and 18 months post implementation of an opioid-prescribing protocol within a federally qualified health center. $\chi^{2}$ and $t$-tests were computed to estimate change in opioid prescribing, morphine-equivalent dose, comedication prescribing, and number and type of protocol elements utilized.

Results: The opioid protocol was associated with an increase in urine drug screens from $18.3 \%$ to $26.8 \%$ from pre to postimplementation $(P<.0001)$. There was no significant increase in opioid treatment agreements. Tramadol $(21.4 \%$ to $16.8 \%, P=.0006)$ and antidepressant $(56.0 \%$ to $51.6 \%, P=$ .012) prescribing significantly decreased. Total opioid prescriptions and maximum morphine-equivalent doses were similar from pre to postimplementation. Protocol elements were more often used when patients had a higher opioid dose and were receiving benzodiazepines.

Conclusions: Implementing a multi-faceted opioid-prescribing protocol was not associated with change in number or dose of opioid prescriptions but was associated with greater use of urine drug screens, and risk reduction tools were used more often in high-risk patients. Implementation research is needed to identify barriers to maximizing adherence to opioid protocols. ( $\mathrm{J}$ Am Board Fam Med 2020;33:27-33.)

Keywords: Antidepressants, Benzodiazepines, Evidence-Based Medicine, Opioids, Pain, Patient Health Questionnaire, Prescriptions, Primary Health Care, Residency, Risk Reduction Behavior, Tramadol

The widespread use of opioid medications to treat chronic noncancer pain has led to a national crisis in the United States, prompting a radical shift in the way primary care physicians are expected to

This article was externally peer reviewed.

Submitted 8 July 2019; revised 25 September 2019; accepted 29 September 2019.

From the Department of Family and Community Medicine, Saint Louis University School of Medicine, St. Louis MO

Funding: This project was supported with Department funds.

Conflict of interest: none declared.

Corresponding author: Matthew Breeden, MD, Saint Louis University School of Medicine, 1402 N. Grand Blvd, St. Louis, MO 63104 (E-mail: Matthew.Breeden@health. slu.edu). manage patients with pain. In 2016 the Centers for Disease Control and Prevention (CDC) released "Guideline for Prescribing Opioids for Chronic Pain,"1 which recommends minimizing the use of opioids for chronic pain, establishing treatment goals with patients, and urine drug screen (UDS) testing both before starting therapy and at least once annually during therapy. This guideline incorporated recommendations from prior groups, many of which were already in various stages of implementation across the country.

Progress in controlling and reducing opioid prescriptions was being made before the release of the Centers for Disease Control and Prevention 
(CDC) guidelines. Analysis of United States prescribing data shows that opioid prescribing peaked in 2010 at a rate of 782 morphine-milligram equivalents (MMEs) per capita, with a decline to 640 MMEs per capita by $2015 .^{2}$ This is still considerably above the 180 MMEs per capita prescribed in 1999. ${ }^{2}$ Despite the prevalence of opioid prescriptions slightly declining over the past few years, the number of fatal and nonfatal opioid overdose deaths continue to rise. ${ }^{3}$ Education of physicians and adherence to guideline concordant practices is urgently needed to improve the safety of opioid prescribing.

In addition to urine drug screening and opioid treatment agreements, validated questionnaires help providers assess patient risk level when opioids are being prescribed. The Opioid Risk Tool (ORT) was designed to identify individuals at risk of "opioid-related, aberrant behaviors" "4 before opiates are prescribed. The 17-item Current Opioid Misuse Measure $(\mathrm{COMM})^{5}$ assesses patients already on long-term opioid therapy, with the goal of identifying patients with high risk of opioid misuse. While these tools are common in clinical use, they have not been validated across the clinical spectrum and evidence is sparse as to their efficacy in reducing opioid misuse. ${ }^{6}$

A randomized controlled trial of 985 primary care patients compared electronic decision support to use of multiple risk reduction strategies, including nurse care management, electronic registries, and provider feedback. ${ }^{7}$ Patients who received multiple risk reduction strategies were more likely to receive guideline-concordant care, including a higher likelihood of opioid treatment agreement and urine drug testing. Those patients were more likely to have dose reduction and opioid treatment discontinuation. Another study investigated the impact of guideline development and dissemination at an academic general internal medicine department. ${ }^{8}$ The guideline for treatment of chronic noncancer pain included quarterly office visits, routine urine drug screening, and use of the Opioid Risk Tool. It resulted in a decrease in patients receiving opioids for chronic noncancer pain and an increase in the use of urine drug screening. Despite the recognized benefit of risk reduction strategies, implementation, and adherence to these strategies, such as urine drug screening and enforcing regular followup for refills, has been poor in primary care. ${ }^{9}$
To support safe opioid prescribing in a family medicine residency, we implemented an opioidprescribing protocol in 2015 to protect patients and providers from abuse and misuse of opioids in the context of chronic pain treatment. The objectives of this study were to 1 ) characterize fidelity to the protocol, 2) determine whether fidelity to the protocol was associated with lower MME prescriptions, and 3) determine whether any 1 component of the prescribing protocol had a stronger association with MMEs prescribed.

\section{Methods}

A committee of faculty and residents developed a protocol for the treatment of chronic noncancer pain for patients at a resident and faculty practice located within a federally qualified health center. The protocol included an Opioid Risk Tool, Urine Drug Screen (UDS) and opioid treatment agreements (OTAs) at the initiation of opioid therapy and the COMM, UDS, and OTAs annually. This protocol was available to all providers as 2 encounter forms, which served as a clinical decision support tool, 1 for an initial visit and 1 for annual followup, within the electronic health record (EHR) that included all relevant elements. The protocol was required for resident visits, all which were precepted by faculty. Faculty were asked to use the protocol, although were not monitored for compliance. At a group meeting before implementation, we educated all faculty and residents about opiate prescription guidelines and the use of the protocol. The protocol was implemented on March 25,2015 . We obtained data from extracted electronic health record data and included demographics; prescription orders (opioids and other concomitant therapies); UDS, ORT, COMM, and OTA dates; Patient Health Questionnaire (PHQ)-9 scores; and body mass index (BMI).

Eligible patients for analysis included pre- (including protocol implementation date) and postimplementation cohorts who had a prescription written for any dose and duration of the following opioid analgesics: codeine, fentanyl, hydrocodone, hydromorphone, methadone, morphine, oxycodone, or oxymorphone. The preimplementation cohort had scripts written from March 25, 2013 to March 25, $2015(\mathrm{n}=1887)$ and the postimplementation cohort from March 26, 2015 through September 26, $2016(\mathrm{n}=1532)$. Data from 2607 patients with 
24,735 opioid prescriptions were extracted during the study period. Some patients $(\mathrm{n}=812)$ overlapped the pre- and postintervention cohorts, but we treated the 2 sampling cohorts independently similar to Chen et al. ${ }^{8}$ Saint Louis University's Institutional Review Board (IRB) approved this study with a waiver of informed consent because the data were obtained from the electronic health record and deidentified after implementing the protocol as a quality improvement project.

\section{Measures}

Protocol Elements

We created binary (yes vs no) variables for protocol elements in the pre- and postintervention period (UDS, ORT, COMM, and OTA). The ORT (first data point: November 11, 2014) and COMM (first data point: February 13, 2015) were not used for the entire preintervention period, thus, we did not create ORT and COMM preintervention variables.

\section{Opioid Fill Information}

We calculated total number of opioid prescriptions per patient in pre- and postintervention cohorts. We calculated morphine-equivalent dose (MED) using standard equianalgesic conversion tables that provide the number of morphine equivalents in milligrams in a given medication, and a maximum daily dose variable per patient for pre- and postimplementation periods. Since a "day's supply" variable was not available, we assumed that prescriptions were for 30 days. If the quantity dispensed was $<30$, daily MED was calculated as the unit dose. If quantity was $\geq 30$, daily MED was (quantity $\times$ unit dose/30). MED calculations excluded methadone prescriptions.

\section{Comedications}

Comedications included the presence of a prescription for the following: tramadol, benzodiazepines, antidepressants, musculoskeletal agents (cyclobenzaprine, chlorzoxazone, methocarbamol, metaxalone, tizanidine, baclofen, carisoprodol, orphenadrine), and anticonvulsants (gabapentin, pregabalin).

\section{Other Variables}

Demographic variables included age, gender, race, Hispanic ethnicity, and marital status. Because depression has a bidirectional association with any prescription opioid use and higher dose and longer duration of use, ${ }^{10,11}$ we characterized the magnitude of depression in our sample to evaluate the potential for depression to bias results. Variables were created for maximum PHQ-9 score and any PHQ-9 score $\geq 10$ (yes vs no). Similarly, obesity is associated with chronic noncancer pain and opioid use. ${ }^{12}$ To assess for potential bias related to obesity or depression, we computed sensitivity analysis in those with at least mild depression defined by a $\mathrm{PHQ} \geq 10$ and separately among patients with obesity defined by $\mathrm{BMI} \geq 30 \mathrm{~kg} / \mathrm{m}^{2}$.

\section{Data Analysis}

We analyzed data using SAS v 9.4 (SAS Institute, Cary, NC) at an $\alpha$ of 0.05 , and summarized data using means $( \pm \mathrm{SD})$ or frequencies and percentages. Because pre- and postimplementation cohorts were treated independently similar to Chen et $\mathrm{al},{ }^{8}$

Table 1. Characteristics of Patients Receiving $\geq 1$ Opioid Prescription from March 25, 2013 to September 25, $2016(n=2607)$

\begin{tabular}{lc}
\hline Characteristic & $\mathrm{n}(\%)$ or Mean $( \pm \mathrm{SD})$ \\
\hline Age, mean $( \pm$ SD) & $45.7( \pm 13.7)$ \\
Gender & \\
Female & $1775(68.1 \%)$ \\
Male & $832(31.9 \%)$ \\
Race & \\
White & $1638(62.8 \%)$ \\
Black & $851(32.6 \%)$ \\
Asian & $56(2.2 \%)$ \\
Other & $55(2.1 \%)$ \\
Unknown & $7(0.3 \%)$ \\
Ethnicity & \\
Hispanic & $70(2.7 \%)$ \\
Non-Hispanic & $2085(80.0 \%)$ \\
Unknown & $452(17.3 \%)$ \\
Marital status & \\
Married & $749(28.7 \%)$ \\
Separated/divorced & $296(11.4 \%)$ \\
Widowed & $143(5.5 \%)$ \\
Single & $1406(53.9 \%)$ \\
Unknown & $13(0.5 \%)$ \\
Any PHQ-9 & $\mathrm{n}=1617$ \\
Max PHQ-9, mean $( \pm S D)$ & $8.6( \pm 7.4)$ \\
Any PHQ $\geq 10$ & $634(39.2 \%)$ \\
Any BMI & $\mathrm{n}=2527$ \\
Max BMI, mean $( \pm S D)$ & $1579(62.5 \%)$ \\
Any BMI $\geq 30$ & \\
\hline
\end{tabular}

BMI, body mass index; PHQ, Patient Health Questionnaire; $\mathrm{SD}$, standard deviations. 
Table 2. Opioid-Prescribing Protocol Elements, Opioid Fill Information, and Co-Medications, for Patients with Any Opioid Prescription in Pre- or Post-Intervention Period

\begin{tabular}{|c|c|c|c|}
\hline & $\begin{array}{l}\text { Pre-Intervention } \\
\quad(\mathrm{n}=1887)\end{array}$ & $\begin{array}{l}\text { Post-Intervention } \\
\quad(\mathrm{n}=1532)\end{array}$ & $P$-Value \\
\hline \multicolumn{4}{|l|}{ Protocol elements } \\
\hline Urine drug screen & $345(18.3 \%)$ & $411(26.8 \%)$ & $<.0001$ \\
\hline ORT completed & $\mathrm{n} / \mathrm{a}$ & $90(5.9 \%)$ & $\mathrm{n} / \mathrm{a}$ \\
\hline COMM & $\mathrm{n} / \mathrm{a}$ & $73(4.8 \%)$ & $\mathrm{n} / \mathrm{a}$ \\
\hline Opioid treatment agreement & $214(11.3 \%)$ & $189(12.3 \%)$ & .369 \\
\hline No. of elements & & & $\mathrm{n} / \mathrm{a}$ \\
\hline 0 & $\mathrm{n} / \mathrm{a}$ & $1080(70.5 \%)$ & \\
\hline 1 & $\mathrm{n} / \mathrm{a}$ & $274(17.9 \%)$ & \\
\hline$\geq 2$ & $\mathrm{n} / \mathrm{a}$ & $178(11.6 \%)$ & \\
\hline No longer on opioid & $1,075(57.0 \%)$ & $\mathrm{n} / \mathrm{a}$ & $\mathrm{n} / \mathrm{a}$ \\
\hline Still on opioid & $812(43.0 \%)$ & $\mathrm{n} / \mathrm{a}$ & $\mathrm{n} / \mathrm{a}$ \\
\hline \multicolumn{4}{|l|}{ Opioid fill information } \\
\hline Total No. RX, mean $( \pm S D)$ & $7.3( \pm 10.2)$ & $7.2( \pm 8.8)$ & .766 \\
\hline \multicolumn{4}{|l|}{ Total No. RX, categories } \\
\hline 1 & $710(37.6 \%)$ & $585(38.2 \%)$ & \\
\hline 2 & $281(14.9 \%)$ & $194(12.7 \%)$ & .273 \\
\hline 3 & $113(6.0 \%)$ & $102(6.7 \%)$ & \\
\hline$\geq 4$ & $783(41.5 \%)$ & $651(42.5 \%)$ & \\
\hline Max daily dose (MED)* & $(\mathrm{n}=1883)$ & $(\mathrm{n}=1527)$ & \\
\hline 1 to $50 \mathrm{mg}$ & $1657(88.0 \%)$ & $1331(87.2 \%)$ & \\
\hline 51 to $100 \mathrm{mg}$ & $176(9.4 \%)$ & $153(10.0 \%)$ & .762 \\
\hline$>100 \mathrm{mg}$ & $50(2.7 \%)$ & $43(2.8 \%)$ & \\
\hline \multicolumn{4}{|l|}{ Co-medications } \\
\hline Tramadol & $404(21.4 \%)$ & $257(16.8 \%)$ & .0006 \\
\hline Benzodiazepines & $489(25.9 \%)$ & $375(24.5 \%)$ & .336 \\
\hline Antidepressants & $1056(56.0 \%)$ & $791(51.6 \%)$ & .012 \\
\hline Musculoskeletal agents & $580(30.7 \%)$ & $453(29.6 \%)$ & .460 \\
\hline Anticonvulsants & $450(23.9 \%)$ & $402(26.2 \%)$ & .108 \\
\hline
\end{tabular}

COMM, current opioid misuse measure; MED, morphine-equivalent dose; ORT, opioid risk tool; RX, prescription; SD, standard deviations.

*Excludes methadone prescriptions.

we assessed differences in the presence of protocol elements, opioid fill information and comedications using $\chi^{2}$ tests for categorical variables and independent samples $t$-tests for continuous variables. We repeated these analyses in sensitivity analysis using subgroups with depression and separately in those with obesity. Similarly, for only the postimplementation cohort, we assessed differences in opioid prescription outcomes and presence of comedications between the presence and absence of each protocol element with $t$-tests and $\chi^{2}$ tests.

\section{Results}

Among the 2607 patients with an opioid prescription from March 25, 2013 to September 25, 2016, average age was $45.7( \pm 13.7)$ years of age, $68.1 \%$ were female, $62.8 \%$ were White, $80.0 \%$ were nonHispanic, and $53.9 \%$ were single. Average PHQ-9 score was $8.6( \pm 7.4)$ and $39.2 \%$ had a score of at least 10. Average BMI was $34.0( \pm 9.2) \mathrm{kg} / \mathrm{m}^{2}$ and $62.5 \%$ were obese (see Table 1 ).

Table 2 shows pre- and postimplementation cohort comparisons. We found an increase in UDS screening from $18.3 \%$ to $26.8 \%$ from pre to postimplementation $(P<.0001)$. OTA rates were similar $(P=.369)$. Total opioid prescriptions and maximum MED were similar from pre to postimplementation. Tramadol (21.4\% to $16.8 \%, P=.0006)$ and antidepressant $(56.0 \%$ to $51.6 \%, P=.012)$ prescribing significantly decreased. 
Results did not change in sensitivity analysis among patients with depression and separately in patients with obesity. However, among patients with depression, a majority remained on opioids (64.2\%); and compared with the sample as a whole, more patients were receiving benzodiazepines before and after the intervention $(37.2 \%$ before and $36.7 \%$ after), see e-Table 1 . In contrast, the distribution of all variables before and after the intervention in those with obesity was nearly the same as in the cohort as a whole (see e-Table 2).

Table 3 shows opioid outcomes and co-medications by each protocol element in the postimplementation cohort. The use of a UDS, COMM, ORT, and OTAs were all positively associated with a greater average number of opioid prescriptions and larger maximum MED. Use of a UDS was significantly associated with benzodiazepine $(P<$ $.0001)$, antidepressant $(P=.0002)$, musculoskeletal agents $(P=.005)$, and anticonvulsant prescriptions $(P=.0002)$. Use of the ORT was significantly associated with greater odds of benzodiazepines $(P=.003)$, antidepressants $(P=.038)$, and musculoskeletal agents $(P=.025)$; use of the COMM was significantly associated with greater odds of a benzodiazepine prescription $(P=.002)$, an antidepressant $(P=.013)$ and anticonvulsants $(P=.003)$. Last, OTAs were significantly associated with greater odds of prescriptions for benzodiazepines $(P=.0007)$, antidepressants $(P<.0001)$, musculoskeletal agents $(P=.0002)$, and anticonvulsants $(P=.0002)$.

\section{Discussion}

When comparing pre- and postintervention cohorts, the implementation of a chronic pain management protocol was associated with increased use of urine drug screens and no change in the use of OTAs. This finding is consistent with other studies designed to improve safe opioid prescribing which reveal increased adoption of urine drug testing but little change in use of other risk reduction strategies. ${ }^{8,9,13}$ We found minimal use of the COMM and ORT in the preintervention cohort, precluding comparison between the pre and post groups. However, we did identify trends in the patients who received these measures in the post-intervention period. Use of the ORT and COMM and OTAs was more likely in patients with multiple opioid prescriptions, higher doses of opioids, and coprescription with either benzodiazepines or antidepressants, the latter 2 medications prescribed much more often among those with depression. Such patients are likely perceived to be at higher risk of misuse, which may influence providers to use risk reduction strategies. Starrels et $\mathrm{al}^{9}$ similarly showed that patients with more opioid misuse factors are more likely to be sent for urine drug testing.

Our results did not show any statistically significant change in the average number of opioid prescriptions or MMEs prescribed after implementing the protocol. These results are partly consistent with Chen and colleagues study ${ }^{8}$ of the effects of implementing an opioid prescribing protocol in an academic primary care practice in 2013 . While we found no change in opioid prescribing, this prior study reported very modest reduction in the percent of patients who received any opioid prescription $(3.9 \%$ to $3.4 \%)$ and the percent of patients on chronic opioids decreased from $2.0 \%$ to $1.6 \%$. As we observed, implementing an opioid protocol seems to have the strongest impact on use of UDS which increased from $9.2 \%$ to $17.3 \%{ }^{8}$

These data highlight the difficulties inherent in change management. The protocol relied on educational sessions and dissemination of protocol documents and was not strictly followed across the practice. This approach does not seem to have made a significant impact in clinic practice. Prior studies have shown similarly low efficacy of such efforts, even when including formal Continuing medical education (CME). ${ }^{14-16} \mathrm{~A}$ recent review suggests that physicians are more likely to respond to educational experiences when they are active and multifaceted, rather than passive and didactic. ${ }^{17}$ One study of opioid prescribing by providers in Washington showed that providers exposed to more intense interventions are more likely to reduce high-dose chronic opioid therapy. ${ }^{18}$ Providers in our health center reacted positively to the implementation of the protocol, and implemented some of the mechanics of the protocol. There appeared to be more consistent use of protocol procedures for patients who had more risk factors for abuse, suggesting that the protocol itself did not change the opinions of providers about the appropriateness of opiate prescriptions for their patients. We believe that to change clinical practice, a more effective intervention may be education about opi- 


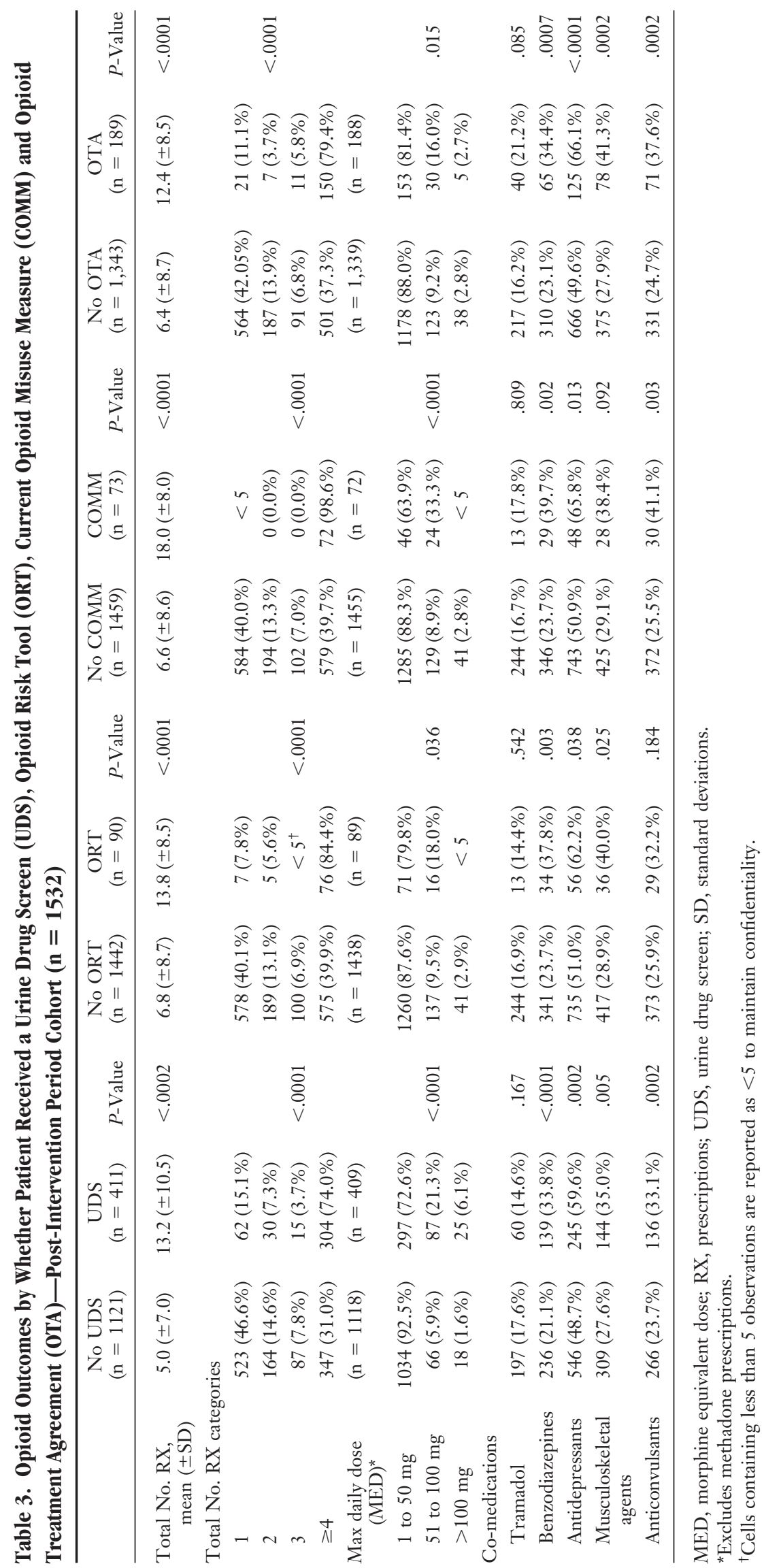


ate addiction and availability of alternate approaches to pain.

\section{Limitations}

Our study was limited by reliance solely on chart documentation and did not include data from pharmacies. However, we feel it is safe to assume that patients receiving numerous opioid prescriptions were likely having them filled. Another limitation of the data are that they were collected at an urban federally qualified health center with a high proportion of low-income patients, limiting generalizability. We were unable to prospectively follow all patients prior and after the protocol was implemented. Therefore, it is possible, though unlikely, that patients treated before implementation systematically differed from those seen after implementation and biased our results.

In summary, our study shows the limitations of implementing a chronic opioid treatment protocol at a faculty and resident clinic. Adherence to the elements of the protocol was low and did not result in a reduction in opioid prescriptions or MMEs prescribed. We observed an increase in the use of urine drug screening and use of risk assessment questionnaires. Given the modest change in opioid prescribing in a prior opioid protocol implemented in academic primary care, ${ }^{8}$ additional studies are needed to identify new prescribing protocol designs that result in safe opioid prescriptions. Future studies should strive for greater levels of participation from providers, and more provider education about appropriate prescribing and other approaches to chronic pain. Implementation research is warranted to identify barriers to full adherence to opioid protocols.

To see this article online, please go to: bttp://jabfm.org/content/ 33/1/27.full.

\section{References}

1. Dowell D, Haegerich TM, Chou R. CDC Guideline for prescribing opioids for chronic pain-United States, 2016. JAMA 2016;315:1624-45.

2. Guy GP Jr, Zhang K, Bohm MK, et al. Vital signs: changes in opioid prescribing in the United States, 2006-2015. MMWR Morb Mortal Wkly Rep 2017; 66:697-704.

3. Rudd RA, Seth P, David F, Scholl L. Increases in drug- and opioid-involved overdose deaths-United States, 2010-2015. MMWR Morb Mortal Wkly Rep 2016;65:1445-52.
4. Webster LR, Webster RM. Predicting aberrant behaviors in opioid-treated patients: preliminary validation of the Opioid Risk Tool. Pain Med 2005;6: 432-42.

5. Butler SF, Budman SH, Fernandez KC, et al. Development and validation of the Current Opioid Misuse Measure. Pain 2007;130:144-56.

6. Webster LR. Risk factors for opioid-use disorder and overdose. Anesth Analg 2017;125:1741-8.

7. Liebschutz JM, Xuan Z, Shanahan CW, et al. Improving adherence to long-term opioid therapy guidelines to reduce opioid misuse in primary care: a cluster-randomized clinical trial. JAMA Intern Med 2017;177:1265-72.

8. Chen JH, Hom J, Richman I, Asch SM, Podchiyska T, Johansen NA. Effect of opioid prescribing guidelines in primary care. Medicine (Baltimore) 2016;95: e4760.

9. Starrels JL, Becker WC, Weiner MG, Li X, Heo M, Turner BJ. Low use of opioid risk reduction strategies in primary care even for high risk patients with chronic pain. J Gen Intern Med 2011;26:958-64.

10. Sullivan MD. Depression effects on long-term prescription opioid use, abuse, and addiction. Clin J Pain 2018;34:878-84.

11. Scherrer JF, Salas J, Copeland LA, et al. Prescription opioid duration, dose, and increased risk of depression in 3 large patient populations. Ann Fam Med 2016;14:54-62.

12. Stokes A, Berry KM, Collins JM, et al. The contribution of obesity to prescription opioid use in the United States. Pain 2019;160:2255-62.

13. Canada RE, DiRocco D, Day S. A better approach to opioid prescribing in primary care. J Fam Practice 2014;63:E1-E8.

14. Sohn W, Ismail AI, Tellez M. Efficacy of educational interventions targeting primary care providers' practice behaviors: an overview of published systematic reviews. J Public Health Dent 2004;64: 164-72.

15. Grimshaw JM, Eccles MP, Walker AE, Thomas RE. Changing physicians' behavior: what works and thoughts on getting more things to work. J Contin Educ Health Prof 2002;22:237-43.

16. Grimshaw JM, Shirran L, Thomas R, et al. Changing provider behavior: an overview of systematic reviews of interventions. Med Care 2001;39:II2II45.

17. Mostofian F, Ruban C, Simunovic N, Bhandari M. Changing physician behavior: what works? Am J Manag Care 2015;21:75-84.

18. Von Korff M, Dublin S, Walker RL, et al. The impact of opioid risk reduction initiatives on highdose opioid prescribing for patients on chronic opioid therapy. J Pain 2016;17:101-10. 
Appendix Table 1. Opioid-Prescribing Protocol Elements, Opioid Fill Information, and Co-Medications, for Patients with a $P H Q \geq 10$ With any Opioid Prescription in Pre- or Post-Intervention Period

\begin{tabular}{|c|c|c|c|}
\hline & $\begin{array}{l}\text { Pre-intervention } \\
\quad(\mathrm{n}=441)\end{array}$ & $\begin{array}{l}\text { Post-intervention } \\
\quad(\mathrm{n}=476)\end{array}$ & $P$-value \\
\hline \multicolumn{4}{|l|}{ Protocol elements } \\
\hline Urine drug screen & $95(21.5 \%)$ & $164(34.5 \%)$ & $<.0001$ \\
\hline ORT completed & $\mathrm{n} / \mathrm{a}$ & $45(9.5 \%)$ & $\mathrm{n} / \mathrm{a}$ \\
\hline COMM & $\mathrm{n} / \mathrm{a}$ & $33(6.9 \%)$ & $\mathrm{n} / \mathrm{a}$ \\
\hline $\begin{array}{l}\text { Opioid Treatment } \\
\text { Agreement }\end{array}$ & $66(15.0 \%)$ & $84(17.7 \%)$ & .273 \\
\hline No. elements & & & $\mathrm{n} / \mathrm{a}$ \\
\hline 0 & $\mathrm{n} / \mathrm{a}$ & $298(62.6 \%)$ & \\
\hline 1 & $\mathrm{n} / \mathrm{a}$ & $93(19.5 \%)$ & \\
\hline$\geq 2$ & $\mathrm{n} / \mathrm{a}$ & $85(17.9 \%)$ & \\
\hline No longer on opioid & $158(35.8 \%)$ & $\mathrm{n} / \mathrm{a}$ & $\mathrm{n} / \mathrm{a}$ \\
\hline Still on opioid & $283(64.2 \%)$ & $\mathrm{n} / \mathrm{a}$ & $\mathrm{n} / \mathrm{a}$ \\
\hline \multicolumn{4}{|l|}{ Opioid fill information } \\
\hline Total No. RX, mean $( \pm S D)$ & $9.2( \pm 11.0)$ & $8.4( \pm 8.9)$ & .217 \\
\hline \multicolumn{4}{|l|}{ Total No. RX, categories } \\
\hline 1 & $128(29.0 \%)$ & $152(31.9 \%)$ & \\
\hline 2 & $60(13.6 \%)$ & $50(10.5 \%)$ & .444 \\
\hline 3 & $22(5.0 \%)$ & $27(5.7 \%)$ & \\
\hline$\geq 4$ & $231(52.4 \%)$ & $247(51.9 \%)$ & \\
\hline Max daily dose (MED)* & $(\mathrm{n}=439)$ & $(\mathrm{n}=474)$ & \\
\hline 1 to $50 \mathrm{mg}$ & $379(86.3 \%)$ & $407(85.9 \%)$ & \\
\hline 51 to $100 \mathrm{mg}$ & $50(11.4 \%)$ & $55(11.6 \%)$ & .963 \\
\hline$>100 \mathrm{mg}$ & $10(2.3 \%)$ & $12(2.5 \%)$ & \\
\hline \multicolumn{4}{|l|}{ Co-medications } \\
\hline Tramadol & $102(23.1 \%)$ & $91(19.1 \%)$ & .136 \\
\hline Benzodiazepines & $164(37.2 \%)$ & $175(36.7 \%)$ & .894 \\
\hline Antidepressants & $335(76.0 \%)$ & $360(75.6 \%)$ & .906 \\
\hline Musculoskeletal agents & $170(38.6 \%)$ & $171(35.9 \%)$ & .411 \\
\hline Anticonvulsants & $136(30.8 \%)$ & $161(33.8 \%)$ & .335 \\
\hline
\end{tabular}

COMM, current opioid misuse measure; MED, morphine-equivalent dose; ORT, opioid risk tool; RX, prescription; SD, standard deviations; PHQ, patient health questionnaire.

*Excludes methadone prescriptions. 
Appendix Table 2. Opioid-Prescribing Protocol Elements, Opioid Fill Information, and Co-Medications, for Patients with a BMI $\geq 30 \mathrm{~kg} / \mathrm{m}^{2}$ with any Opioid Prescription in Pre- or Post-Intervention Period

\begin{tabular}{|c|c|c|c|}
\hline & $\begin{array}{l}\text { Pre-Intervention } \\
\quad(\mathrm{n}=1161)\end{array}$ & $\begin{array}{l}\text { Post-Intervention } \\
\quad(\mathrm{n}=963)\end{array}$ & $P$-Value \\
\hline \multicolumn{4}{|l|}{ Protocol elements } \\
\hline Urine drug screen & $202(17.4 \%)$ & $251(26.1 \%)$ & $<.0001$ \\
\hline ORT completed & $\mathrm{n} / \mathrm{a}$ & $60(6.2 \%)$ & $\mathrm{n} / \mathrm{a}$ \\
\hline COMM & $\mathrm{n} / \mathrm{a}$ & $49(5.1 \%)$ & $\mathrm{n} / \mathrm{a}$ \\
\hline $\begin{array}{l}\text { Opioid treatment } \\
\text { agreement }\end{array}$ & $125(10.8 \%)$ & $127(13.2 \%)$ & .086 \\
\hline No. elements & & & $\mathrm{n} / \mathrm{a}$ \\
\hline 0 & $\mathrm{n} / \mathrm{a}$ & $680(70.6 \%)$ & \\
\hline 1 & $\mathrm{n} / \mathrm{a}$ & $169(17.6 \%)$ & \\
\hline$\geq 2$ & $\mathrm{n} / \mathrm{a}$ & $114(11.8 \%)$ & \\
\hline No longer on opioid & $616(53.1 \%)$ & $\mathrm{n} / \mathrm{a}$ & $\mathrm{n} / \mathrm{a}$ \\
\hline Still on opioid & $545(46.9 \%)$ & $\mathrm{n} / \mathrm{a}$ & $\mathrm{n} / \mathrm{a}$ \\
\hline \multicolumn{4}{|l|}{ Opioid fill information } \\
\hline Total No. RX, mean ( \pm SD) & $7.7( \pm 10.6)$ & $7.6( \pm 9.0)$ & .811 \\
\hline \multicolumn{4}{|l|}{ Total No. RX, categories } \\
\hline 1 & $406(35.0 \%)$ & $341(35.4 \%)$ & \\
\hline 2 & $176(15.2 \%)$ & $118(12.3 \%)$ & .274 \\
\hline 3 & $72(6.2 \%)$ & $64(6.6 \%)$ & \\
\hline$\geq 4$ & $507(43.7 \%)$ & $440(45.7 \%)$ & \\
\hline Max daily dose $(\mathrm{MED})^{*}$ & $(\mathrm{n}=1158)$ & $(\mathrm{n}=958)$ & \\
\hline 1 to $50 \mathrm{mg}$ & $1016(87.7 \%)$ & $822(85.8 \%)$ & \\
\hline 51 to $100 \mathrm{mg}$ & $112(9.7 \%)$ & $109(11.4 \%)$ & .409 \\
\hline$>100 \mathrm{mg}$ & $30(2.6 \%)$ & $27(2.8 \%)$ & \\
\hline \multicolumn{4}{|l|}{ Co-medications } \\
\hline Tramadol & $265(22.8 \%)$ & $182(18.9 \%)$ & .027 \\
\hline Benzodiazepines & $298(25.7 \%)$ & $247(25.7 \%)$ & .992 \\
\hline Antidepressants & $685(59.0 \%)$ & $534(55.5 \%)$ & .100 \\
\hline Musculoskeletal agents & $386(33.3 \%)$ & $321(33.3 \%)$ & .967 \\
\hline Anticonvulsants & $310(26.7 \%)$ & $287(29.8 \%)$ & .113 \\
\hline
\end{tabular}

COMM, current opioid misuse measure; MED, morphine equivalent dose; ORT, opioid risk tool; RX, prescription; SD, standard deviations; BMI, body mass index.

*Excludes methadone prescriptions. 